\title{
GI8. SERVIÇOS: PAPEL DA DIVISÃO DE ATENDIMENTO AO CLIENTE NA CONSTRUÇÃO DE UMA IMAGEM POSITIVA DE BIO-MANGUINHOS JUNTO À HEMORREDE NAT.
}

Linda Khalili Boukai ${ }^{1}$; Ludmila Nascimento Rocha Villar Bezerra ${ }^{1}$; Priscilla Caroline Almeida dos Santos ${ }^{1}$; Marilúcia Sobrado Pina ${ }^{1}$; Tatiana Rose Pizzani Trindade ${ }^{1}$; Vivian Rabello; Adriana Rodrigues Pedro ${ }^{1}$; Beatriz Coutinho Brum $^{1}$; Alessandra Bógio ${ }^{1}$; Denise Maria Lobo Crivelli²

${ }^{1}$ Departamento de Relações com o Mercado, Instituto de Tecnologia em Imunobiológicos, Fundação Oswaldo Cruz.

${ }^{2}$ Divisão de Atendimento ao Cliente e Pós-Marketing, Departamento de Relações com o Mercado, Instituto de Tecnologia em Imunobiológicos, Fundação Oswaldo Cruz.

INTRODUÇÃO A palavra "serviço" tem o significado de "ato ou efeito de servir" e constitui uma modalidade de atendimento presente, de forma cada vez mais significativa, no panorama do mercado contemporâneo. A prestação de serviços é peça fundamental no processo de crescimento e maturação da relação empresa-cliente, influenciando diretamente na apuração dograu de satisfação, ferramenta de indicador de qualidade. A prestação de serviços eficientes, com impactos positivos, contribui favoravelmente para a gestão da organização e com a consolidação da sua imagem.

OBjetIvo Apresentar a atuação da Divisão de Atendimento ao Cliente e Pós-Marketing de Bio-Manguinhos (DIACM) na área de Serviços, com ênfase na qualidade do suporte técnico-científico prestado à Hemorrede NAT. Apontaros pontos-chave na ótica da satisfação dos clientes e no alcance das metas no que tange aocumprimento dos requisitos de atendimento. Destacar a relação com as empresas parceiras no atendimento e a prática de cinco anos da relação contratual.

METODOLOGIA A DIACM é o canal de relacionamento com o cliente, responsável pela prestação de serviços de assistência técnico-científica ao campo. As bases da prestação de serviços à Hemorrede estão apoiadas em duas vertentes principais: suporte científico ao kit NAT HIV / HCV / HBV e suporte técnico à plataforma de equipamentos que operam interfaceados para emissão dos resultados. A equipe de atendimento,amplamente treinada, opera com procedimentos padronizados balizados pelos contratos de assistência técnica com as três empresas fabricantes dos equipamentos que compõem a plataforma NAT - QIAGEN, PerkinElmer e ThermoFisher. 
RESULTADO A prestação de serviços pela DIACM é umtrabalhode equipe dedicado que agrega valores ao público destinatário, via de regra, o cidadão brasileiro que recebe uma doação de sangue seguro e confiável. A importância deste trabalho se reflete no total de 10.229.944bolsas testadas (2011-2015) e na apuração de mais de 50 janelas imunológicas detectadas, evitando a contaminação direta de mais de 200 pessoas. Desde 2011, a DIACM prestou 11.651 atendimentos, retornando respostas com qualidade. A apuração do grau de satisfação revelou que $88 \%$ dos clientes estão satisfeitos com os serviços prestados pela DIACM. Em relação às empresas parceiras, a QIAGEN possui $85 \%$ de satisfação, PerkinElmer $82 \%$ e ThermoFisher $81 \%$. Os serviços prestados pela DIACM também incluem aplicação de pesquisas para avaliação das condições de entrega dos produtos (kit e acessórios). Até o momento, mais de 1.000 pesquisas foram aplicadas com o intuito de buscar oportunidades de melhorias no serviço.

CONCLUSÃo O sucesso de uma organização pública requer a conquista de resultados positivos em consonância com sua missão institucional. Neste cenário, Bio-Manguinhos ganha destaque pelos altos índices de satisfaçãoda Hemorrede, apurados nas pesquisas, enfatizando a satisfaçãodos clientes com a qualidade dos serviços prestados. O paradigma mudou: a unidade de valor, hoje, além do produto, é uma prestação de serviços eficiente e eficaz.

PALAVRAS-CHAVE serviço, atendimento ao cliente, pesquisa de satisfação. 